\title{
Sensitivity of Marginal Maximum Likelihood Estimation of Item and Ability Parameters to the Characteristics of the Prior Ability Distributions
}

\author{
Tae-Je Seong \\ Ewha Womans University
}

The sensitivity of marginal maximum likelihood estimation of item and ability $(\theta)$ parameters was examined when the prior $\theta$ distributions are not matched to the underlying $\theta$ distributions. Thirty sets of 45 -item test data were generated by specification of three types of underlying $\theta$ distributions. They were then analyzed with PC-BILOG. Appropriate specification of the prior $\theta$ distribution increased the accuracy of estimation for item and $\theta$ parameters when the sample size was large. With a small dataset, the appropriate specification of the prior increased the accuracy of $\theta$ parameter estimation, but it did not have that effect on item parameter esti- mation. Only with a large dataset and matched underlying and prior $\theta$ distributions did increasing the number of quadrature points improve the accuracy of estimation of the item parameters. However, the accuracy of $\theta$ estimation was increased by increasing the number of quadrature points, regardless of sample size and appropriateness of the prior $\theta$ distribution. The number of examinees had an important effect on the accuracy of item parameter estimation. Index terms: ability distribution, BILOG, item response theory, marginal maximum likelihood estimation, parameter estimation, quadrature points.

Birnbaum's (1968) joint estimation paradigm has been the standard procedure for maximum likelihood estimation (MLE) of item and ability $(\theta)$ parameters. His joint maximum likelihood estimation (JMLE) method uses an iterative two-stage "back and forth" procedure for estimating item and $\theta$ parameters. This approach was implemented in the widely used LOGIST computer program (Wingersky, Barton, \& Lord, 1982; Wingersky \& Lord, 1973).

Neyman and Scott (1948) pointed out an inherent problem of MLE: Large numbers of incidental parameters affect the consistency of the estimates of the structural parameters. In the context of JMLE, item discrimination $(\alpha)$ and item difficulty $(\beta)$ are structural parameters that are fixed in number by the size of the test. The examinees' $\theta$ s are the incidental parameters, the number of which depends on the sample size. This problem of JMLE was discussed in the context of item response theory (IRT) by Hambleton and Swaminathan (1985, pp. 127-129).

As an alternative to JMLE estimation, the structural parameters can be estimated if the likelihood function can be expressed without any reference to the incidental parameters. Bock and Lieberman (1970) proposed marginal maximum likelihood estimation (MMLE) to remove the effect of incidental parameters by integrating them over the $\theta$ distribution. MMLE estimates item and $\theta$ parameters in separate stages without a "back and forth" scheme; yet, this approach poses a formidable computational task and is practical for only very short tests.

In order to solve the computational problem, Bock and Aitkin (1981) used a modification of the EM algorithm formulated by Dempster, Laird, and Rubin (1977). One characteristic of this MMLE/EM approach is the use of Bayesian concepts, such as prior and posterior distributions. The MMLE/EM 
approach of Bock and Aitkin has been implemented in the BILOG computer program (Mislevy \& Bock, 1982, 1984, 1986).

Because of the difficulty of direct integration with a digital computer, MMLE employs a numerical analysis technique to integrate examinees' $\theta$ s over the $\theta$ distribution. BILOG has an option that allows the user to specify the number of quadrature points, values of quadrature points, and weights corresponding to each quadrature point.

Several item-recovery studies (Drasgow, 1989; Mislevy \& Stocking, 1989; Qualls \& Ansley, 1985; Yen, 1987) have investigated the accuracy of MMLE/EM for item and $\theta$ parameter estimation. Basically, accuracy was defined by how far the item and $\theta$ estimates were from their underlying parameters, and how these estimates were correlated with these parameters.

Qualls and Ansley (1985), Yen (1987), and Mislevy and Stocking (1989) compared LOGIST and BILOG results. Qualls and Ansley found that BILOG uniformly took more computer time to produce estimates than did LOGIST, but the BILOG estimates were uniformly more accurate than the LOGIST estimates.

Yen (1987) compared BILOG (version 2.2) and LOGIST 5.0 (version 2.5) in estimating item and $\theta$ parameters, item response functions (IRF), and test response functions (TRF) for the three-parameter logistic model. Simulated data were generated under a three-parameter model for one 10-item test, four 20 -item tests, and four 40 -item tests for 1,000 examinees from a normal $(\mu=0, \sigma=1) \theta$ distribution, and 20 - and 40 -item reading vocabulary tests for 1,000 examinees from three different nonnormal $\theta$ distributions. Yen concluded that BILOG almost always produced more accurate estimates of individual underlying item parameters. In estimating $\theta$, BILOG was more accurate for the 10-item test, and the two programs were about equally accurate for the 20 -item tests with normal true $\theta$ distribution. However, LOGIST almost always produced more accurate $\theta$ estimates for the 40 -item tests and the tests with non-normal $\theta$ distributions. For the IRF, TRF, and true scores, BILOG gave more accurate estimates than LOGIST for the 10-item test, and both were nearly equally accurate for the 20 - and 40 -item tests.

Mislevy and Stocking (1989) found that LOGIST and BILOG recovered the true item parameter estimates for a 45-item test equally well, and that BILOG estimated item parameters more accurately than LOGIST for a 15 -item test. Bayesian expected a posteriori (EAP) in BILOG estimated the $\theta$ parameters more accurately than LOGIST. The study recommended that a user with a short test and a small examinee sample should consider using BILOG. They also indicated that both programs provide similar item and $\theta$ parameter estimates for longer tests and large samples, and that LOGIST might be preferred on the basis of computing costs.

Drasgow (1989) evaluated MMLE for the two-parameter logistic model in estimating item parameters, standard errors (SE) of item parameters, and IRFs. Four test lengths were used. The numbers of items were $5,10,15$, and 25 , with sample sizes of $200,300,500$, and 1,000. He found that MMLE was substantially more accurate than JMLE for item parameter estimation, and that the estimated SES of JMLE were far less accurate than the Fletcher-Powell SEs of MMLE. For IRFS, MMLE provided accurate recovery for much shorter tests and scales than JMLE.

The above recovery studies concluded that the BILOG estimates generally were more accurate than the LOGIST estimates for short tests and small numbers of examinees. However, none of the abovementioned studies specified different prior $\theta$ distributions or other factors associated with these distributions; only normal prior $\theta$ distributions were used. The characteristics of the prior $\theta$ distribution affect the posterior distribution of $\theta$ used in estimating item parameters, and if the prior $\theta$ distribution is correctly matched with the underlying $\theta$ distribution, MMLE has produced consistent estimates of item parameters (Harwell, Baker, \& Zwarts, 1988). Thus, the agreement of the specified 
prior and underlying $\theta$ distributions needs to be considered; the goal of this research was to investigate the role of the prior $\theta$ distribution in parameter estimation under the MMLE/EM approach.

\section{Method}

\section{Factors}

The four factors that may affect the item and $\theta$ parameter estimates in the marginal maximum likelihood procedure, and that were varied in this study, are

1. The type of prior $\theta$ distribution (normal, positively- and negatively-skewed);

2. The number of quadrature points $(10,20)$;

3. The type of underlying $\theta$ distribution (normal, positively- and negatively-skewed); and

4. The number of examinees $(100,1,000)$.

In educational applications of IRT, latent $\theta$ distributions are not always normal, especially in populations with specific characteristics. The positively-skewed distribution for the present study was defined by a $\chi^{2}$ distribution having eight degrees of freedom $(d f)$, because the skewness of this distribution is 1 . The equation of the positively-skewed distribution is

$f(x)=\frac{1}{2^{4} \Gamma(4)} x^{3} e^{-x / 2}$.

This equation has a mean of 8 and a standard deviation of 4 . The $\theta$ scores were standardized $(0,1)$. The negatively-skewed distribution was simply the mirror image of the positively-skewed distribution. Figure 1 shows the three types of $\theta$ distributions. Discrete approximations to the $\theta$ distributions were used, both for generating item response vectors and as prior distributions when estimating parameters.

Figure 1

The Three Types of Prior Ability Distributions

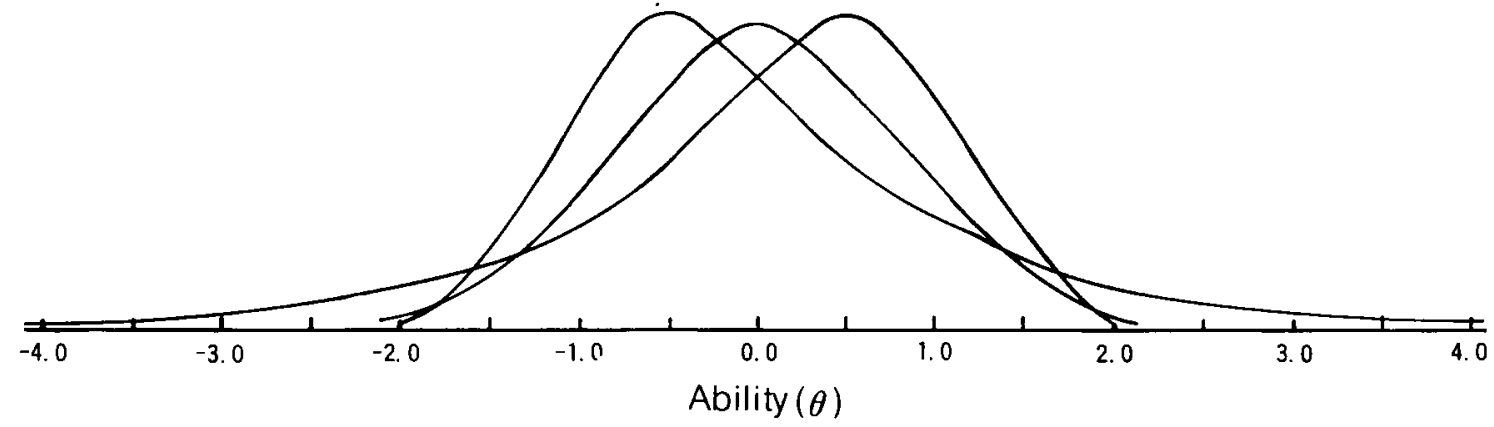

BILOG uses 10 or 20 quadrature points for parameter estimation as default values, based on whether the number of items in a test is less or more than 50 . Therefore, the present study used these two numbers of quadrature points.

Generally, the MMLE/EM approach is more accurate than the JMLE method when the sample size is small, and both approaches are very similar when the sample size is large. For example, Drasgow (1989) found that the accuracy of MMLE was increased by increasing sample size. The present study used a small sample (100 examinees) and a large sample (1,000 examinees). The number of items in a test was not of interest as a factor in the design of the experiment; test length in psychological and educational applications commonly ranges from 20 to 50 items. A test of 45 items was used here because this number of items was large enough to yield stable results. 


\section{Item Response Data Generation (GENIRV Runs)}

Simulated item response vectors $(1,0)$ were generated by GENIRV (Baker, 1978). This program requires the user to specify the number of items in a test, the values of item parameters, the sample size, and the type of underlying $\theta$ distribution. For each specification set, five replications of the item response data were generated using different seeds for the random-number generator. In total, $30 \mathrm{GENIRV}$ runs were performed. Item discrimination parameters were randomly selected from within each cell of Table 1 for the 45 items generating item response vectors under the two-parameter normal ogive IRT model.

Table 1

Number of Items at Each Level of Item Difficulty $(\beta)$ and for Three Ranges of Item Discrimination $(\alpha)$

\begin{tabular}{lcccc}
\hline \hline \multirow{2}{*}{$\begin{array}{c}\text { Range } \\
\text { of } \alpha\end{array}$} & \multicolumn{4}{c}{ Item Difficulty $(\beta)$} \\
\cline { 2 - 5 } .3 to.5 & -1.0 & 0.0 & +1.0 & Total \\
.6 to.8 & 5 & 5 & 5 & 15 \\
.9 to 1.1 & 5 & 5 & 5 & 15 \\
Total & 15 & 15 & 5 & 15 \\
\hline
\end{tabular}

The underlying $\theta$ distribution was implemented using the number of $\theta$ groups, $\theta$ levels of the groups, and number of examinees in each $\theta$ group. These values were based on the given underlying $\theta$ distribution. Thirteen $\theta$ groups were used for the 100 -examinee data, and $31 \theta$ groups were used for the 1,000-examinee data, because these groupings and numbers of examinees yielded a good match to the desired skewed and normal underlying $\theta$ distributions.

\section{Item and $\theta$ Parameter Estimation (BILOG Runs)}

Each of the generated datasets was analyzed with the microcomputer version of BILOG (PC-BILOG version 1.1; Mislevy \& Bock, 1986), and the three types of prior $\theta$ distributions, as well as the two numbers of quadrature points, were specified. Most of the program default values were used, except the specification for the prior $\theta$ distribution, which was defined by the number of quadrature points, their values, and the weights that corresponded to each quadrature point. The values and weights under the given prior $\theta$ distribution were computed by the graphical solution (Mislevy's histogram solution) used in BILOG.

For item parameter estimation, user-supplied quadrature points and weights for the prior $\theta$ distribution were used. Option 2 in the IDIstp command in the calibration part was used to designate the type of prior $\theta$ distribution for all specifications, except for the normal prior $\theta$ distribution and 10 quadrature points (which result from the default option). A two-parameter normal ogive metric was also selected.

The default lognormal ( $\mu=.0, \sigma=.5$ ) prior distributions of the item discrimination parameters were used. In addition, the means of these prior distributions were estimated from the data by specifying the "Float" command in BILOG runs; the effect of using floating priors is that all parameters of a given type shrink toward the mean of that type with a predetermined strength, while that mean is estimated from the data (see Mislevy, 1986; Mislevy \& Stocking, 1989). No prior was employed in the estimation of the item difficulty parameters.

For $\theta$ estimation, Bayesian EAP was selected because no other estimator has smaller mean squared 
error over the population for which the distribution of $\theta$ is specified by the prior (Bock \& Mislevy, 1982; Mislevy \& Stocking, 1989). In order to investigate the effect of the number of quadrature points, the same number of quadrature points and weights were used in both item and $\theta$ parameter estimation.

\section{Measures of Accuracy}

Two descriptive statistics were used to assess the accuracy of the MMLE/EM approach. The first was the square root of the mean squared difference between the estimates and parameters-that is, the root mean square error (RMSE) (Yen, 1987). The second was the mean of the absolute difference between estimates and parameters. This bias measure reflects how far the item and $\theta$ parameter estimates are from the known values (Qualls \& Ansley, 1985).

\section{Analysis of Experimental Design}

In addition to obtaining difference measures, a split-plot factorial design was employed to investigate the effects of the types of the prior $(p)$ and underlying $(u) \theta$ distributions, the number of quadrature points $(q)$, and the number of examinees $(e)$. The between-block factors were the type of underlying $\theta$ distribution and the number of examinees. The within-block factors were the type of prior distribution and the number of quadrature points.

The dependent variables employed in the ANOVA were the log of the average of the squared difference between estimates, and the parameters for item difficulty, item discrimination, and $\theta$. These were called log mean square errors (LMSE); the LMSE for item discrimination estimates was defined as:

LMSE $=\log \frac{1}{J} \sum_{j=1}^{J}\left(\hat{\boldsymbol{\alpha}}_{j}-\alpha_{j}\right)^{2}$.

Comparisons were performed to check the effect of pairing the prior $\theta$ distribution with the given underlying $\theta$ distribution. The non-orthogonal contrasts for the effect of prior $\theta$ distribution with the given underlying distribution were:

$\psi_{1}=\mu_{\mathrm{N}}-\mu_{+}$

for the normal underlying $\theta$ distribution,

$\psi_{2}=\mu_{-}-\mu_{\mathrm{N}}$

for the negatively-skewed underlying $\theta$ distribution,

$\psi_{3}=\mu_{-}-\mu_{+}$

for the negatively-skewed underlying $\theta$ distribution,

$\psi_{4}=\mu_{+}-\mu_{\mathrm{N}}$

for the positively-skewed underlying $\theta$ distribution, and

$\psi_{s}=\mu_{+}-\mu_{-}$

for the positively-skewed underlying $\theta$ distribution,

where $\mu_{\mathrm{N}}$ denotes a normal prior $\theta$ distribution,

$\mu_{+}$denotes a positively-skewed prior $\theta$ distribution, and

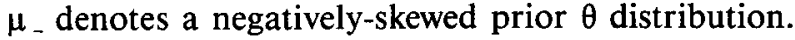

The contrast for the overall effect of matched or nonmatched underlying prior $\theta$ distributions was

$\psi_{6}=\frac{1}{3}\left(\mu_{\mathrm{NN}}+\mu_{--}+\mu_{++}\right)-\left[\frac{1}{6}\left(\mu_{\mathrm{N}-}+\mu_{\mathrm{N}_{+}}+\mu_{-\mathrm{N}}+\mu_{-+}+\mu_{+\mathrm{N}}+\mu_{+-}\right)\right]$,

where the first subscript denotes the underlying $\theta$ distribution and the second subscript denotes the prior $\theta$ distribution. 
The first five contrasts show the effect of matched or nonmatched prior $\theta$ distribution conditional on each given underlying $\theta$ distribution. The last contrast shows the effect of the matched cases versus the nonmatched cases over all $\theta$ distributions.

\section{Results}

\section{Item Discrimination Estimates}

The average RMSE and average bias measures for item discrimination estimates are reported in Table 2. The overall means of the RMSE and bias measure for item discrimination estimates were 146 and .112. The averages of the RMSE and bias measures for all matched cases were .121 and .095 , whereas those of the RMSE and bias measures for all nonmatched cases were .159 and .120. In the matched cases, the averages of the RMSE and bias measures for 100 examinees were .179 and .138 , whereas the average measures for 1,000 examinees were .064 and .052 . The averages of the RMSE for 10 and 20 quadrature points were .147 and .146; the average bias measures were .112 and .111. The averages of the RMSE for 100 and 1,000 examinees were .196 and .097 ; the average bias measures were .149 and .074 .

\section{Table 2}

Root Mean Square Error (RMSE) and Bias for Item

Discrimination Estimates $(\alpha)$ for Normal, Negative, and Positive True $\theta$ Distributions, $N=100$ and 1,000 Examinees, and 10 and 20 Quadrature Points

\begin{tabular}{|c|c|c|c|c|c|c|}
\hline \multirow{3}{*}{$\begin{array}{c}\text { Distribution, } \\
N \text {, and } \\
\text { Statistic }\end{array}$} & \multicolumn{6}{|c|}{ Prior $\theta$ Distribution } \\
\hline & \multicolumn{2}{|c|}{ Normal } & \multicolumn{2}{|c|}{ Negative } & \multicolumn{2}{|c|}{ Positive } \\
\hline & 10 & 20 & 10 & 20 & 10 & 20 \\
\hline \multicolumn{7}{|c|}{$\begin{array}{l}\text { Normal } \theta \text { Distribution } \\
\qquad N=100\end{array}$} \\
\hline RMSE & .189 & .184 & .204 & .208 & .207 & .217 \\
\hline Bias & .146 & .140 & .151 & .153 & .157 & .163 \\
\hline \multicolumn{7}{|l|}{$N=1,000$} \\
\hline RMSE & .072 & .066 & .107 & .111 & .117 & .121 \\
\hline Bias & .059 & .052 & .079 & .083 & .085 & .087 \\
\hline \multirow{2}{*}{\multicolumn{7}{|c|}{$\begin{array}{l}\text { Negative } \theta \text { Distribution } \\
\qquad N=100\end{array}$}} \\
\hline & 173 & 173 & & 175 & 248 & \\
\hline $\begin{array}{l}\text { RIMSE } \\
\text { Bias }\end{array}$ & .131 & .132 & .170 & .115 & $\begin{array}{l}.248 \\
187\end{array}$ & .235 \\
\hline \multicolumn{7}{|l|}{$N=1,000$} \\
\hline RMSE & .089 & .072 & .065 & .065 & .136 & .146 \\
\hline Bias & .070 & .056 & .051 & .051 & .103 & .112 \\
\hline \multicolumn{7}{|c|}{$\begin{array}{l}\text { Positive } \theta \text { Distribution } \\
N=100\end{array}$} \\
\hline RMSE & .177 & .177 & .217 & .222 & .172 & .176 \\
\hline Bias & .139 & .136 & .165 & .168 & .136 & .138 \\
\hline \multicolumn{7}{|l|}{$N=1,000$} \\
\hline RMSE & .090 & .072 & .146 & .153 & .060 & .060 \\
\hline Bias & .071 & .056 & .111 & .118 & .049 & .048 \\
\hline
\end{tabular}

The average difference measures of the matched distributions were generally smaller than those of the nonmatched cases for each underlying $\theta$ distribution. The largest average difference measures were obtained in the nonmatched case of the negatively- and positively-skewed $\theta$ distributions. 
Increasing the number of quadrature points generally reduced the average difference measures when the underlying $\theta$ distribution was matched to the prior $\theta$ distribution. As might be expected, the average difference measures were decreased by increasing the sample size for each given underlying and prior $\theta$ distribution and number of quadrature points.

The results obtained from performing the analysis of variance of the split-plot factorial design of the LMSE for item discrimination estimates are summarized in Table 3. Among the between-blocks effects, only the examinee effect was significant at an $\alpha$ level of .05 . The $\eta^{2}$ value of .642 indicates that much of the total variability in the item discrimination estimates was due to the effect of the number of examinees. Among the within-blocks effects, most of the effects were significant at an $\alpha=.05$. The main effect of the number of quadrature points was significant.

Table 3

Results of the Analysis of Variance of Log Mean Square Errors (LMSE) for Item Discrimination Estimates

\begin{tabular}{|c|c|c|c|c|c|}
\hline Source & $d f$ & SS & MS & $F$ & $\eta^{2}$ \\
\hline Between Blocks & 29 & & & & \\
\hline$u$ & 2 & .591 & .295 & .73 & \\
\hline$e$ & 1 & 100.346 & 100.346 & $247.96^{*}$ & .642 \\
\hline ue & 2 & .029 & .014 & .04 & \\
\hline Block Within ue & 24 & 9.712 & .405 & Error I & \\
\hline Within Blocks & 150 & & & & \\
\hline$p$ & 2 & 6.936 & & & .044 \\
\hline$p u$ & 4 & 26.585 & & & .170 \\
\hline$\psi_{1}$ & 1 & 4.880 & 4.880 & $136.65^{*}$ & \\
\hline$\psi_{2}$ & 1 & .413 & 413 & $11.53^{*}$ & \\
\hline$\psi_{3}$ & 1 & 12.010 & 12.010 & $335.56^{*}$ & \\
\hline$\Psi_{4}$ & 1 & .941 & .941 & $26.29 *$ & \\
\hline$\Psi_{s}$ & 1 & 13.455 & 13.455 & $375.29^{*}$ & \\
\hline$\psi_{6}$ & 1 & 18.000 & 18.000 & $502.94^{*}$ & \\
\hline pe & 2 & 1.056 & .528 & $14.76^{*}$ & .006 \\
\hline pue & 4 & 8.166 & 2.042 & $57.04^{*}$ & .052 \\
\hline$p$ Block Within $u e$ & 48 & 1.718 & 0.036 & Error II & \\
\hline$q$ & 1 & .056 & .056 & $7.68^{*}$ & .0004 \\
\hline$q u$ & 2 & .051 & .025 & $3.49^{*}$ & .0003 \\
\hline$q e$ & 1 & .064 & .064 & $8.80^{*}$ & .0004 \\
\hline que & 2 & .017 & .008 & 1.15 & \\
\hline$q$ Block Within ue & 24 & .175 & .007 & Error III & \\
\hline$p q$ & 2 & .551 & .276 & $40.32^{*}$ & .004 \\
\hline$p q u$ & 4 & .033 & .008 & 1.20 & \\
\hline pqe & 2 & .370 & .185 & $27.08^{*}$ & .002 \\
\hline pque & 4 & .097 & .024 & $3.55^{*}$ & .001 \\
\hline$p q$ Block Within $u e$ & 48 & .328 & .007 & Error IV & \\
\hline Total & 179 & 156.348 & & & \\
\hline
\end{tabular}

Note: $F(.05 ; 1,24)=4.26 ; F(.05 ; 2,24)=3.40 ; F(.05 ; 2,48)=3.20$; $F(.05 ; 4,48)=2.58$.

All six non-orthogonal contrasts of interest for item discrimination estimates were also significant. The critical value for the contrasts was 6.690. Rejecting the null hypotheses of the first five contrasts suggests that the average LMSE of using matched prior $\theta$ distributions was different from those of the nonmatched prior $\theta$ distribution under each underlying $\theta$ distribution. Rejecting the last hypothesis suggests that the average LMSE of the matched prior $\theta$ distribution was different from 
that of the nonmatched prior $\theta$ distribution over all underlying $\theta$ distributions.

The $\eta^{2}$ of the interaction effect between the prior and the underlying $\theta$ distributions was .170 , which means that $17 \%$ of the total sum of squares of the LMSE for item discrimination estimates was due to the interaction effect between two $\theta$ distributions. In order of $\eta^{2}$ values, the most influential effects were the examinee effect $(64.2 \%)$, and the two-way interaction effect $(17.0 \%)$ between the prior and the underlying $\theta$ distribution.

\section{Item Difficulty Estimates}

The average RMSE and average bias measures for item difficulty estimates are reported in Table 4. The overall means of the RMSE and bias measure for item difficulty estimates were .200 and .155 . The averages of the RMSE and bias measures for all matched cases were .193 and .149 , whereas those of the RMSE and bias measures for all nonmatched cases were .204 and .159. In the matched cases of two $\theta$ distributions, the averages of the RMSE and bias measures for 100 examinees were .280 and .217 , whereas they were .106 and .081 for 1,000 examinees. The averages of the RMSE for 10 and 20 quadrature points were .204 and .197 ; the average bias measures were .159 and .152 . The averages of the RMSE with 100 and 1,000 examinees were .279 and .122 ; the average bias measures were .215 and .096 .

\section{Table 4}

RMSE and Bias for Item Difficulty Estimates ( $\beta$ ) for Normal, Negative, and Positive True $\theta$ Distributions, $N=100$ and 1,000 Examinees, and 10 and 20 Quadrature Points

\begin{tabular}{|c|c|c|c|c|c|c|}
\hline \multirow{3}{*}{$\begin{array}{c}\text { Distribution, } \\
N \text {, and } \\
\text { Statistic }\end{array}$} & \multicolumn{6}{|c|}{ Prior $\theta$ Distribution } \\
\hline & \multicolumn{2}{|c|}{ Normal } & \multicolumn{2}{|c|}{ Negative } & \multicolumn{2}{|c|}{ Positive } \\
\hline & 10 & 20 & 10 & 20 & 10 & 20 \\
\hline \multicolumn{7}{|c|}{ Normal $\theta$ Distribution } \\
\hline RMSE & .301 & .285 & .281 & .281 & .281 & .281 \\
\hline Bias & .238 & .221 & .216 & .217 & .220 & .220 \\
\hline \multicolumn{7}{|l|}{$N=1,000$} \\
\hline RMSE & .108 & .101 & .130 & .128 & .129 & .125 \\
\hline Bias & .085 & .076 & .102 & .101 & .103 & .101 \\
\hline \multicolumn{7}{|c|}{$\begin{array}{l}\text { Negative } \theta \text { Distribution } \\
N=100\end{array}$} \\
\hline RMSE & .303 & .262 & .265 & .261 & .251 & .249 \\
\hline Bias & .229 & .200 & .202 & .198 & .200 & .190 \\
\hline \multicolumn{7}{|l|}{$N=1,000$} \\
\hline RMSE & .123 & .103 & .104 & .103 & .135 & .134 \\
\hline Bias & .096 & .079 & .079 & .077 & .107 & .107 \\
\hline \multicolumn{7}{|c|}{$\begin{array}{l}\text { Positive } \theta \text { Distribution } \\
\quad N=100\end{array}$} \\
\hline RMSE & .307 & .289 & .275 & .275 & .288 & .281 \\
\hline Bias & .234 & .222 & .213 & .213 & .224 & .218 \\
\hline \multicolumn{7}{|l|}{$N=1,000$} \\
\hline RMSE & .123 & .110 & .161 & .159 & .111 & .110 \\
\hline Bias & .097 & .086 & .127 & .130 & .086 & .086 \\
\hline
\end{tabular}

The average difference measures of the matched distributions were generally smaller than those of the nonmatched cases for each given underlying $\theta$ distribution only with the 1,000 examinees. 
The largest average difference measures were obtained in the nonmatched case involving the negativelyand the positively-skewed $\theta$ distributions. Increasing the number of quadrature points decreased the average difference measures when the prior $\theta$ distributions were matched to the underlying $\theta$ distributions. The average difference measures were decreased by increasing the sample size in all combinations of prior and underlying $\theta$ distributions and the number of quadrature points.

The results obtained from performing the analysis of variance of the split-plot factorial design for the LMSE of item difficulty estimates are summarized in Table 5. Among the between-blocks effects, only the effect of the number of examinees was significant at $\alpha=.05$. The $\eta^{2}$ value of .901 shows that $90.1 \%$ of the total sum of squares of the LMSE for the item difficulty estimates was due to the effect of the number of examinees. Among the within-blocks effects, the main effect of the number of quadrature points was significant at $\alpha=.05$. Only the fourth of the non-orthogonal contrasts was not rejected. The critical value for the contrasts was 6.690. Almost all of the total sum of squares of the LMSE for item difficulty estimates $(96.1 \%)$ was due to the effect of the number of examinees.

\section{$\theta$ Estimates}

The average RMSE and average bias measures for $\theta$ estimates are reported in Table 6 . The grand

Table 5

Results of the Analysis of Variance of LMSEs for Item Difficulty Estimates

\begin{tabular}{|c|c|c|c|c|c|}
\hline Source & $d f$ & SS & MS & $F$ & $\eta^{2}$ \\
\hline Between Blocks & 29 & & & & \\
\hline$u$ & 2 & .824 & .412 & 2.36 & \\
\hline$e$ & 1 & 124.908 & 124.908 & $716.98^{*}$ & .901 \\
\hline ue & 2 & .131 & .066 & .38 & \\
\hline Block Within ue & 24 & 4.181 & .174 & Error I & \\
\hline Within Blocks & 150 & & & & \\
\hline$p$ & 2 & .234 & & & .002 \\
\hline$p u$ & 4 & 1.809 & & & .013 \\
\hline$\Psi_{1}$ & 1 & .238 & .238 & $8.94^{*}$ & \\
\hline$\Psi_{2}$ & 1 & .215 & .215 & $8.08^{*}$ & \\
\hline$\Psi_{3}$ & 1 & .485 & .485 & $18.25^{*}$ & \\
\hline$\Psi_{4}$ & 1 & .009 & .009 & .33 & \\
\hline$\psi_{s}$ & 1 & 1.112 & 1.112 & $41.81^{*}$ & \\
\hline$\psi_{6}$ & 1 & 1.391 & 1.391 & $52.30^{*}$ & \\
\hline$p e$ & 2 & 1.517 & .759 & $28.53^{*}$ & .011 \\
\hline pue & 4 & 2.588 & .647 & $24.33^{*}$ & .018 \\
\hline$p$ Block Within $u e$ & 48 & 1.276 & .027 & Error II & \\
\hline$q$ & 1 & .330 & .330 & $48.61^{*}$ & .002 \\
\hline$q u$ & 2 & .048 & .024 & $3.55^{*}$ & .0003 \\
\hline$q e$ & 1 & .010 & .010 & $1.46^{*}$ & \\
\hline que & 2 & .003 & .001 & .22 & \\
\hline$q$ Block Within $u e$ & 24 & .163 & .007 & Error III & \\
\hline$p q$ & 2 & .323 & .161 & $45.64^{*}$ & .002 \\
\hline$p q u$ & 4 & .068 & .017 & 4.78 & .001 \\
\hline pqe & 2 & .013 & .007 & 1.88 & \\
\hline pque & 4 & .010 & .003 & 0.73 & \\
\hline$p q$ Block Within $u e$ & 48 & .170 & .004 & Error IV & \\
\hline Total & 179 & 138.605 & & & \\
\hline
\end{tabular}

Note: $F(.05 ; 1,24)=4.26 ; F(.05 ; 2,24)=3.40 ; F(.05 ; 2,48)=3.20$; $F(.05 ; 4,48)=2.58$. 
means of the RMSE and bias measures over all datasets were .323 and .250 . The average of the RMSE and bias measures for all matched cases were .294 and .234, whereas those of the RMSE and bias measures for all nonmatched cases were .338 and .258 . The averages of the RMSE for 10 and 20 quadrature points were .332 and .314 ; the average bias measures were .256 and .244 . The averages of the RMSE for 100 and 1,000 examinees were .318 and .329; the average bias measures were .248 and .251 . The average accuracy measures for $\theta$ estimates were not changed drastically by changing the number of examinees, which occurred with item discrimination and item difficulty estimates.

Table 6

RMSE and Bias for $\theta$ Estimates for Normal, Negative, and Positive True $\theta$ Distributions, $N=100$ and 1,000

Examinees, and 10 and 20 Quadrature Points

\begin{tabular}{|c|c|c|c|c|c|c|}
\hline \multirow{3}{*}{$\begin{array}{c}\text { Distribution, } \\
N \text {, and } \\
\text { Statistic }\end{array}$} & \multicolumn{6}{|c|}{ Prior $\theta$ Distribution } \\
\hline & \multicolumn{2}{|c|}{ Normal } & \multicolumn{2}{|c|}{ Negative } & \multicolumn{2}{|c|}{ Positive } \\
\hline & 10 & 20 & 10 & 20 & 10 & 20 \\
\hline \multicolumn{7}{|c|}{$\begin{array}{l}\text { Normal } \theta \text { Distribution } \\
N=100\end{array}$} \\
\hline RMSE & .316 & .292 & .335 & .315 & .326 & .309 \\
\hline Bias & .252 & .233 & .260 & .247 & .256 & .243 \\
\hline \multicolumn{7}{|l|}{$N=1,000$} \\
\hline RMSE & .312 & .292 & .336 & .323 & .335 & .322 \\
\hline Bias & .248 & .232 & .256 & .250 & .257 & .249 \\
\hline \multicolumn{7}{|c|}{ Negative $\theta$ Distribution } \\
\hline RMSE & .338 & .294 & .290 & .281 & .372 & .346 \\
\hline Bias & .270 & .235 & .233 & .225 & .277 & .261 \\
\hline \multicolumn{7}{|l|}{$N=1,000$} \\
\hline RMSE & .324 & .306 & .299 & .294 & .405 & .382 \\
\hline Bias & .258 & .244 & .236 & .233 & .284 & .275 \\
\hline \multicolumn{7}{|c|}{$\begin{array}{l}\text { Positive } \theta \text { Distribution } \\
N=100\end{array}$} \\
\hline RMSE & .323 & .299 & .364 & .346 & .288 & .283 \\
\hline Bias & .257 & .237 & .269 & .260 & .229 & .227 \\
\hline \multicolumn{7}{|l|}{$N=1,000$} \\
\hline RMSE & .321 & .302 & .404 & .382 & .290 & .288 \\
\hline Bias & .254 & .238 & .280 & .273 & .230 & .227 \\
\hline
\end{tabular}

Given each underlying $\theta$ distribution, the average difference measures of the matched prior $\theta$ distribution were smaller than those of the two nonmatched cases in both sample sizes. When the skewed prior $\theta$ distributions were not matched with the skewed underlying $\theta$ distributions, large average difference measures were obtained in both sample sizes. The average difference measures were decreased by increasing the number of quadrature points in all combinations of given underlying distributions, prior distributions, and sample sizes. The range of the average difference measures for $\theta$ estimates was relatively small.

The results obtained from performing the analysis of variance of the split-plot factorial design of the LMSE for $\theta$ estimates are summarized in Table 7. Among the between-blocks effects, only the effect of the number of examinees was significant at $\alpha=.05$. However, the $\eta^{2}$ value of .024 shows that only a small amount of the total variability in the $\theta$ estimates was due to the effect of the number of examinees. Among the within-blocks effects, most of the effects were significant 
at $\alpha=.05$. The main effect of the number of quadrature points was significant, and all six contrasts of interest for $\theta$ estimates were significant (the critical value for the contrasts was 6.690). The sum of $\eta^{2}$ of the interaction effect between the prior and the underlying $\theta$ distributions was .653. Hence, $65.3 \%$ of the total sum of squares of the LMSE for $\theta$ estimates was due to the interaction effect between two $\theta$ distributions. In order of $\eta^{2}$ values, the most influential effects were the effect of the interaction between prior and underlying $\theta$ distributions, the effect of the number of quadrature points, and the effect of the prior $\theta$ distribution.

Table 7

Results of the Analysis of Variance of LMSEs for $\theta$ Estimates

\begin{tabular}{|c|c|c|c|c|c|}
\hline Source & $\overline{d f}$ & SS & MS & $\bar{F}$ & $\overline{\eta^{2}}$ \\
\hline Between Blocks & 29 & & & & \\
\hline$u$ & 2 & .079 & .040 & 1.15 & \\
\hline$e$ & 1 & .199 & .199 & $5.75^{*}$ & .024 \\
\hline ue & 2 & .028 & .014 & 0.40 & \\
\hline Block Within ue & 24 & .829 & .035 & Error I & \\
\hline Within Blocks & 150 & & & & \\
\hline$p$ & 2 & .519 & & & .063 \\
\hline pu & 4 & 5.387 & & & .653 \\
\hline$\psi_{1}$ & 1 & .157 & .157 & $35.58^{*}$ & \\
\hline$\psi_{2}$ & 1 & .255 & .255 & $57.59 *$ & \\
\hline$\psi_{3}$ & 1 & 2.619 & 2.619 & $592.46^{*}$ & \\
\hline$\psi_{4}$ & 1 & .253 & .253 & $57.24^{*}$ & \\
\hline$\psi_{5}$ & 1 & 2.751 & 2.751 & $622.38^{*}$ & \\
\hline$\psi_{6}$ & 1 & 2.972 & 2.972 & $672.31^{*}$ & \\
\hline pe & 2 & .096 & .048 & $10.82^{*}$ & .012 \\
\hline pue & 4 & .122 & .031 & $6.90^{*}$ & .015 \\
\hline$p$ Block Within ue & 48 & .212 & .004 & Error II & \\
\hline$q$ & 1 & .544 & .544 & $325.72 *$ & .066 \\
\hline$q u$. & 2 & .009 & .005 & 2.68 & \\
\hline$q e$ & 1 & .019 & .019 & $11.32^{*}$ & .002 \\
\hline que & 2 & .007 & .004 & 2.22 & \\
\hline$q$ Block Within $u e$ & 24 & .041 & .002 & Error III & \\
\hline$p q$ & 2 & .058 & .029 & $27.39 *$ & .007 \\
\hline$p q u$ & 4 & .040 & .010 & $9.42^{*}$ & .005 \\
\hline$p q e$ & 2 & .006 & .003 & 2.82 & \\
\hline pque & 4 & .010 & .003 & 2.34 & \\
\hline$p q$ Block Within $u e$ & 48 & .051 & .001 & Error IV & \\
\hline Total & 179 & 8.255 & & & \\
\hline
\end{tabular}

\section{Conclusions}

Matching or not matching between underlying and prior $\theta$ distributions is an important consideration when item and $\theta$ parameters are estimated with MMLE/EM. It was shown that item discrimination and difficulty parameters were estimated more accurately when the two $\theta$ distributions were matched and sample size was large. $\theta$ estimation with MMLE/EM showed clearer results than did item parameter estimation. Even with small datasets, examinees' $\theta$ s were estimated more precisely when the type of prior $\theta$ distribution was specified correctly. More than half of the total sum of squares of the LMSE for $\theta$ estimation was due to the specification of the prior $\theta$ distribution.

Only with a large dataset and matched underlying and prior $\theta$ distributions, did increasing the 
number of quadrature points improve the accuracy of estimation of the item parameters. The accuracy of $\theta$ estimation was improved by increasing the number of quadrature points, regardless of sample size and appropriateness of the prior $\theta$ distribution.

As expected, increasing sample size improved the accuracy of estimation for the item discrimination and item difficulty parameters. The average difference measures (RMSE and bias) for 1,000 examinees were one-half of those for 100 examinees. Increasing the number of examinees for $\theta$ estimation did not improve the accuracy of $\theta$ estimation; this reflects the fact that $\theta$ is estimated one examinee at a time, regardless of sample size.

The results suggest that when a user is estimating item and $\theta$ parameters with BILOG, it is important to consider the match between the type of underlying and prior $\theta$ distributions employed and the number of quadrature points. For item parameter estimation with large datasets, users should specify the type of prior $\theta$ distribution from either theoretical or empirical considerations. When users do not have an idea about the type of prior $\theta$ distribution, it is better to use the default normal prior $\theta$ distribution than to have chosen an inappropriate prior $\theta$ distribution. With small sample sizes, it is not necessary to designate the correct type of prior $\theta$ distribution, as the default normal prior $\theta$ distribution is a safe choice. The number of quadrature points for item parameter estimation is important in the case of matched $\theta$ distributions with large sample sizes. When users are able to specify the appropriate type of prior $\theta$ distribution and have a large sample size, they should increase the number of quadrature points; otherwise, it is safe to use the program defaults for the prior $\theta$ distribution and the number of quadrature points.

For $\theta$ estimation with BILOG, users should specify an appropriate prior $\theta$ distribution and increase the number of quadrature points for $\theta$ estimation, even with small datasets when they know the type of prior $\theta$ distribution to use. When they do not know the type of prior $\theta$ distribution, it is more conservative to use the default normal prior $\theta$ distribution than to have selected an inappropriate prior $\theta$ distribution.

The present study found inconsistent results for item parameter estimation with small datasets. When the negatively-skewed underlying $\theta$ distribution with 100 examinees was matched with a negatively-skewed prior $\theta$ distribution, the average difference measures for item discrimination estimation were larger than when it was paired with a normal prior $\theta$ distribution. Some unexpected results were also found for item difficulty estimates with 100 examinees. Given each underlying $\theta$ distribution, the average difference measures in cases of the matched distribution were larger than those of the nonmatched $\theta$ distributions. The above illogical results may be related to the small sample size.

\section{References}

Baker, F. B. (1978). GENIRV: Computer program for generating item responses. Unpublished manuscript, University of Wisconsin, Madison.

Birnbaum, A. (1968). Some latent trait models and their use in inferring an examinee's ability. In F. M. Lord \& M. R. Novick (Eds.), Statistical theories of mental test scores (pp. 397-472). Reading MA: Addison-Wesley.

Bock, R. D., \& Aitkin, M. (1981). Marginal maximum likelihood estimation of item parameters: An application of an EM algorithm. Psychometrika, 46, 443-459.

Bock, R. D., \& Lieberman, M. (1970). Fitting a re- sponse model for $n$ dichotomously scored items. Psychometrika, 35, 179-197.

Bock, R. D., \& Mislevy, R. J. (1982). Adaptive EAP estimation of ability in a microcomputer environment. Applied Psychological Measurement, 6 , 431-444.

Dempster, A. P., Laird, N. M., \& Rubin, D. B. (1977). Maximum likelihood for incomplete data via the EM algorithm. Journal of the Royal Statistical Society, Series B, 39, 1-38.

Drasgow, F. (1989). An evaluation of marginal maximum likelihood estimation for the two-parameter logistic model. Applied Psychological Measurement, 
$13,77-90$.

Hambleton, R. K., \& Swaminathan, H. (1985). Item response theory: Principles and application. Boston: Kluwer-Nijhoff.

Harwell, M. R., Baker, F. B., \& Zwarts, M. (1988). Item parameter estimation via marginal maximum likelihood and an EM algorithm. A didactic. Journal of Educational Statistics, 13, 243-271.

Mislevy, R. J. (1986). Bayes modal estimation in item response models. Psychometrika, 51, 177-195.

Mislevy, R. J., \& Bock, R. D. (1982). BILOG: Maximum likelihood item analysis and test scoring with logistic models for binary items. Chicago: International Educational Services.

Mislevy, R. J., \& Bock, R. D. (1984). BILOG I maximum likelihood item analysis and test scoring: Logistic model. Mooresville IN: Scientific Software, Inc.

Mislevy, R. J., \& Bock, R. D. (1986). PC-BILOG: Item analysis and test scoring with binary logistic models. Mooresville IN: Scientific Software, Inc.

Mislevy, R. J., \& Stocking, M. L. (1989). A consumer's guide to LOGIST and BILOG. Applied Psychological Measurement, 13, 57-75.

Neyman, J., \& Scott, E. L. (1948). Consistent estimates based on partially consistent observation. Econometrika, 16, 1-32.

Qualls, A. L., \& Ansley, T. N. (1985). A comparison of item and ability parameter estimates derived from LOGIST and BILOG. Paper presented at the meeting of the National Council on Measurement in Education, Chicago IL, U.S.A.

Wingersky, M. S., Barton, M. A., \& Lord, F. M. (1982). LOGIST user's guide. Princeton NJ: Educational Testing Service.

Wingersky, M. S., \& Lord, F. M. (1973). A computer program for estimating examinee ability and item characteristic curve parameters when there are omitted response (Research Memorandum RM-73-2). Princeton NJ: Educational Testing Service.

Yen, M. Y. (1987). A comparison of the efficiency and accuracy of BILOG and LOGIST. Psychometrika, 52, 275-291.

\section{Author's Address}

Send requests for reprints or further information to Tae-Je Seong, School of Education, Ewha Womans University, Seoul, 120-750, Korea. 